\title{
Height-Gain for VLF Radio Waves
}

\author{
James R. Wait and Kenneth P. Spies \\ Contribution from Central Radio Propagation Laboratory, \\ National Bureau of Standards, Boulder, Colo.
}

(Received September 24, 1962)

\begin{abstract}
The height dependence of the field strength of VLF radio waves is considered. Using previously developed theory, the height-gain function of the first two modes is calculated in terms of Airy functions of complex argument. It is indicated, for frequencies of the order of 25 kilocycles per second, that the height-gain function reaches a maximum value at a height of the order of 40 kilometers when the reflecting layer is about 70 kilometers. The form of the height-gain function is also shown to be dependent on the finite conductivity of the ground. An experimental curve for 18.0 kilocycles per second based on a rocket measurement shows some agreement with the theory.
\end{abstract}

\section{Introduction}

Despite the extensive literature [Budden, 1962; Wait, 1962, etc.] on the propagation of VLF radio waves hardly any attention has been paid to the influence of varying the heights of the transmitting or receiving antennas. Although the theory is usually developed for arbitrary heights of the source and observer, the explicit calculations are usually carried out only when both heights are set equal to zero. Because of the long wavelength involved it is expected that the heights of the terminal points would need to be very great before any significant change is felt. However, with the use of high altitude rockets, field strengths can be measured right up to the ionosphere and, thus, the calculations are more than just of academic interest.

It is the purpose of this paper to describe the results of some computations which enable the height-gain function to be plotted for the range from the ground up to the reflecting layers. To simplify the calculations the ionosphere is regarded as a sharply bounded medium. The excitation factors of the various modes are also discussed since they are closely related to the height-gain functions. The significance of the excitation factor itself has been discussed recently [Wait, 1962] where some explicit results were given and the curves of the excitation factor given here extend these somewhat.

\section{Formulation and Statement of Formulas}

At the risk of appearing repetitious, the formulation of the problem and the final results are briefly stated here. The source is a vertical electric dipole located on or above the surface of a smooth spherical earth of radius $a$, conductivity $\sigma_{g}$, and dielectric constant $\epsilon_{g}$. Spherical coordinates $(r, \theta, \phi)$ are chosen with the dipole located at $r=a+z_{0}$ and $\theta=0$. The ionosphere is represented as an isotropic reflecting shell located at $r=a+h$. The electrical proper- ties of this shell are not specified except to say that the tangential electric and magnetic fields are related at the level $r=a+h$ by a surface impedance $Z$.

For harmonic time dependence, the radial component of the resultant electric field is written

$$
E_{\tau} \cong \frac{e^{-i k a \theta}}{a(\theta \sin \theta)^{\frac{1}{2}}} V e^{+i \omega t}
$$

apart from a constant factor. An expression for $V$ was derived previously [Wait, 1961] as the sum of waveguide modes. It may be written conveniently in the following form

$$
V=\frac{4(\pi x)^{\frac{1}{2}}}{y_{0}} e^{-i \pi / 4} \sum e^{-i x t} n G_{n}(\hat{y}) G_{n}(y) \Lambda_{n},
$$

where $x=(k a / 2)^{\frac{1}{3}} \theta, \quad \hat{y}=(2 / k a)^{\frac{1}{3}} k z_{0}, \quad$ and $y=(2 / k a)^{\frac{1}{3}}$ $k(r-a)$. The other factors in this equation are discussed below.

The complex values $t_{n}$ are solutions of the equation

where

$$
1-A(t) B(t)=0,
$$

$$
\begin{gathered}
A(t)=-\left[\frac{w_{1}^{\prime}\left(t-y_{0}\right)+q_{i} w_{1}\left(t-y_{0}\right)}{w_{2}^{\prime}\left(t-y_{0}\right)+q_{i} w_{2}\left(t-y_{0}\right)}\right], \\
B(t)=-\left[\frac{w_{2}^{\prime}(t)-q w_{2}(t)}{w_{1}^{\prime}(t)-q w_{1}(t)}\right], \\
q_{i}=-i(k a / 2)^{\frac{1}{3}} Z / \eta_{0}, \quad \eta_{0}=120 \pi, \\
q \simeq-i(k a / 2)^{\frac{1}{3}}\left(\frac{i \epsilon_{0} \omega}{\sigma+i \epsilon \omega}\right)^{\frac{1}{2}} \text { and } y_{0}=(2 / k a)^{\frac{1}{3}} k h .
\end{gathered}
$$

The functions $w_{1}(t)$ and $w_{2}(t)$ are Airy integral functions and the primes indicate a derivative with respect to the argument. Methods used to obtain numerical values of $t_{n}$ are described in a technical note [Spies and Wait, 1961]. 
The functions $G_{n}$ are height-gain functions and they are normalized to unity for $y$ or $y_{0}$ equal to zero. Explicitly,

where

$$
G_{n}(y)=\frac{f\left(t_{n}, y\right)}{f\left(t_{n}, 0\right)}
$$

$$
f\left(t_{n}, y\right)=w_{1}\left(t_{n}-y\right)+A\left(t_{n}\right) w_{2}\left(t_{n}-y\right) .
$$

The function $\Lambda_{n}$ is a modal excitation factor [Wait, 1961]. Here it is normalized such that, in the limit of zero curvature and perfect ground conductivity, it becomes unity for all modes. Under this condition

$$
\Lambda_{n}=\frac{y_{0}}{2}\left[\left(t_{n}-q^{2}\right)-\frac{\left(t_{n}-y_{0}-q_{i}^{2}\right)\left[w_{2}^{\prime}\left(t_{n}\right)-q w_{2}\left(t_{n}\right)\right]^{2}}{\left[w_{2}^{\prime}\left(t_{n}-y_{0}\right)+q_{i} w_{2}\left(t_{n}-y_{0}\right]^{2}\right.}\right]^{-1} .
$$

In many applications at VLF the ionosphere may be described in terms of an effective conductivity parameter $\omega_{r}$. On neglecting the terrestrial magnetic field and assuming that $\omega<<\nu$ where $\nu$ is the collision frequency, it follows that $\omega_{r}=\omega_{0}^{2} / \nu$ where $\omega_{0}$ is the (angular) plasma frequency. Then, to within a good approximation, the surface impedance is given by [Wait, 1962]

$$
\frac{\eta_{0}}{Z} \cong\left(1+\frac{\omega_{r}}{i \omega}\right)\left(\frac{i \omega}{\omega_{r}}\right)^{\frac{1}{2}}
$$

being essentially independent of angle of incidence (or mode number) for grazing modes.

\section{Excitation Factor}

The excitation factor $\Lambda_{n}$ determines the efficiency of launching of a given mode in the earth ionosphere waveguide. Using (8) and the known values of $t_{n}$ [Spies and Wait, 1961], calculations of $\Lambda_{n}$ were carried out. The resulting curves of $\Lambda_{1}$ and $\Lambda_{2}$ are shown in figures $1 \mathrm{a}$ and $1 \mathrm{~b}$ for $\sigma_{g}=\infty$ corresponding to perfectly conducting ground. To simplify matters even further, $q_{i}$ is taken to be $\infty$ which corresponds to a perfectly reflecting ionosphere with a local reflection coefficient of -1 . It is immediately evident from these curves that the excitation of the least attenuated mode becomes very weak at the higher frequencies. However, for the range of frequencies and heights shown, the second mode is being well excited. In fact, there may be a $2 \mathrm{db}$ increase over the excitation amplitude for a flat earth.

For the cases shown in figures $1 \mathrm{a}$ and $1 \mathrm{~b}, \Lambda_{n}$ is real. Actually, finite ground conductivity or losses in the ionospheric reflecting layer will modify $\Lambda_{n}$ slightly and cause it to become complex. To illustrate this phenomenon, $\Lambda_{1}$ is calculated for various values of $\sigma_{g}$ for an $\omega_{r}$ value of $2 \times 10^{5}$ which is considered to be typical for daytime conditions. The results are shown in figures $2 \mathrm{a}$ and $2 \mathrm{~b}$. It is immediately evident, on comparing the curve for $\sigma_{g}=\infty$ with the corresponding curve for $h=70 \mathrm{~km}$
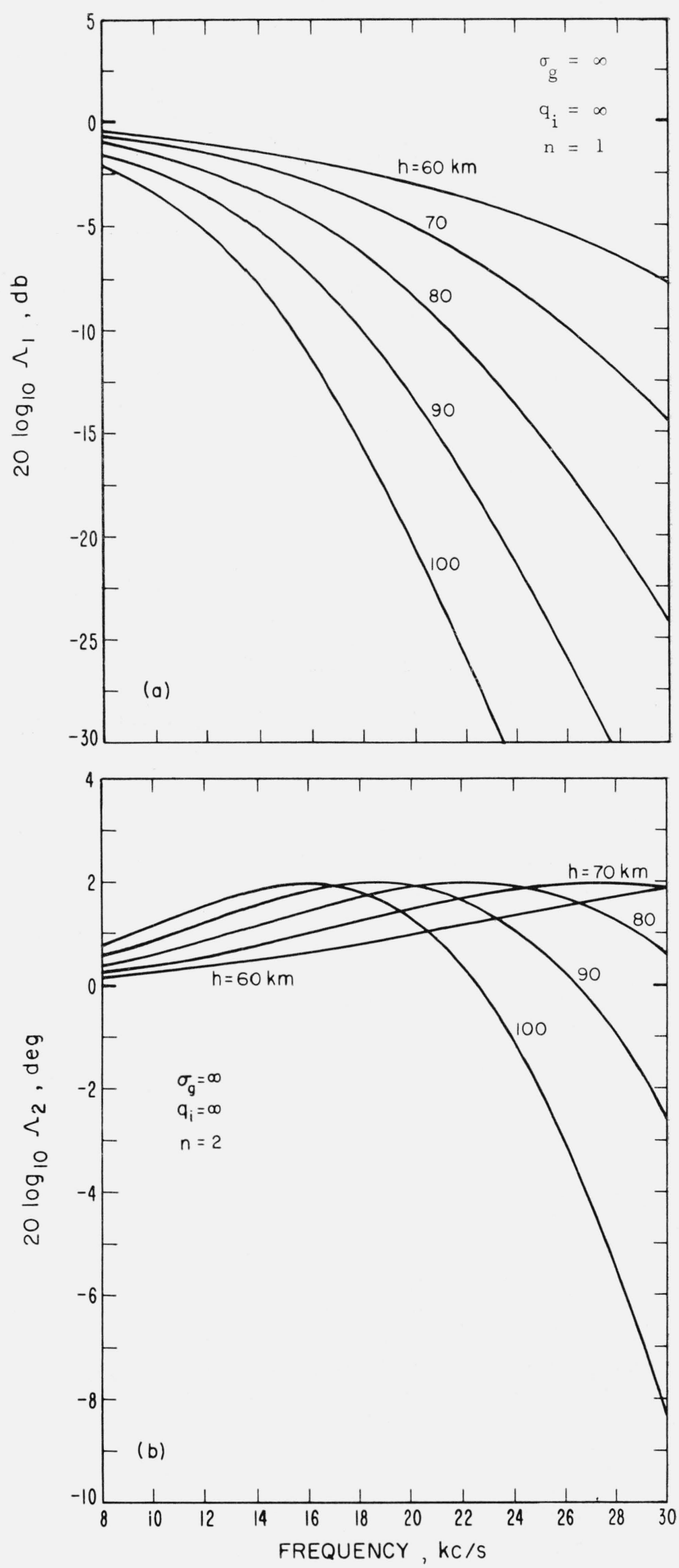

Figures 1a and 1b. The excitation factor for the first and second order modes for an earth-ionosphere waveguide with perfectly reflecting boundaries.

In this case $\Lambda_{n}$ is real.

in figure 1a, that the influence of the finite value of $\omega_{r}$ is quite small. However, the effect of finite ground conductivity is quite noticeable. It is rather interesting to see that the excitation factor 

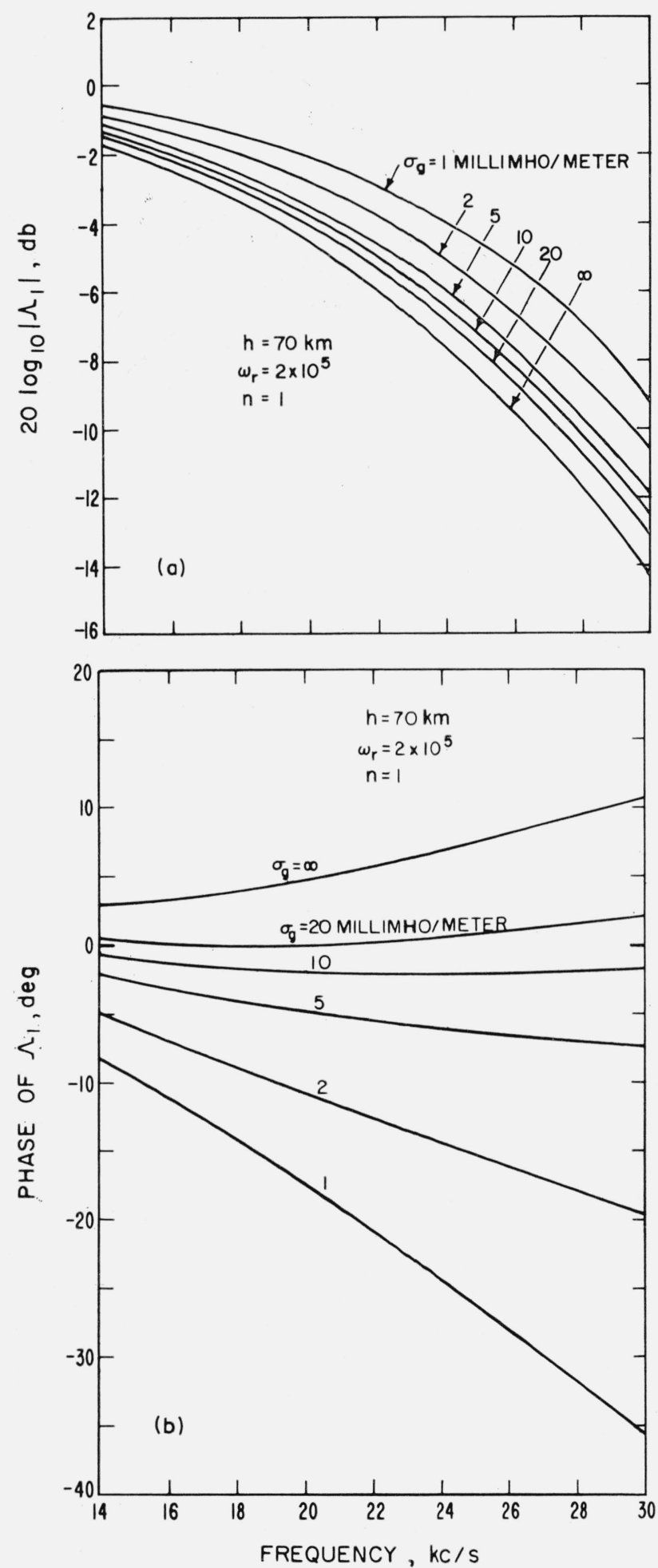

Figures $2 \mathrm{a}$ and $2 \mathrm{~b}$. The (complex) excitation factor for the first-order mode for imperfectly reflecting boundaries.

is actually increased as the ground conductivity is decreased. This fact is not incompatible with the behavior of the height-gain functions as discussed below.

\section{Height-Gain Functions}

The height-gain factors $G_{n}(\hat{y})$ and $G_{n}(y)$ have the same functional form and, therefore, only one of these quantities need be considered. The factors $G_{n}(y)$ may be computed either directly from (6) or use may be made of power series developments [Wait, 1962]. For the results given here, the former method was employed.

The results are shown in figures $3 \mathrm{a}$ to $6 \mathrm{~b}$ with all relevant parameters being indicated on the curves. In figures $3 \mathrm{a}, 3 \mathrm{~b}, 4 \mathrm{a}$, and $4 \mathrm{~b}, G_{n}(y)$ is real since both the ground and the ionosphere are perfectly reflecting. However, when either $\omega_{r}$ or $\sigma_{g}$ is finite, $G_{n}(y)$ is complex as indicated in figures $5 \mathrm{a}, 5 \mathrm{~b}, 6 \mathrm{a}$, and $6 \mathrm{~b}$.

The sets of curves in figures $3 \mathrm{a}$ and $3 \mathrm{~b}$ are for a fixed height $h$ of $70 \mathrm{~km}$ and, because $q_{i}=\infty$, the value of $G_{n}(y)$ must go to zero at $z=70 \mathrm{~km}$ for all the frequencies. A similar behavior is evident for figures $4 \mathrm{a}$ and $4 \mathrm{~b}$ but here the ionosphere heights are taken to be $60,70,80,90$, and $100 \mathrm{~km}$ in turn. When $\omega_{r}$ is finite it can be seen from figures 5 and 6 that $G_{n}(y)$

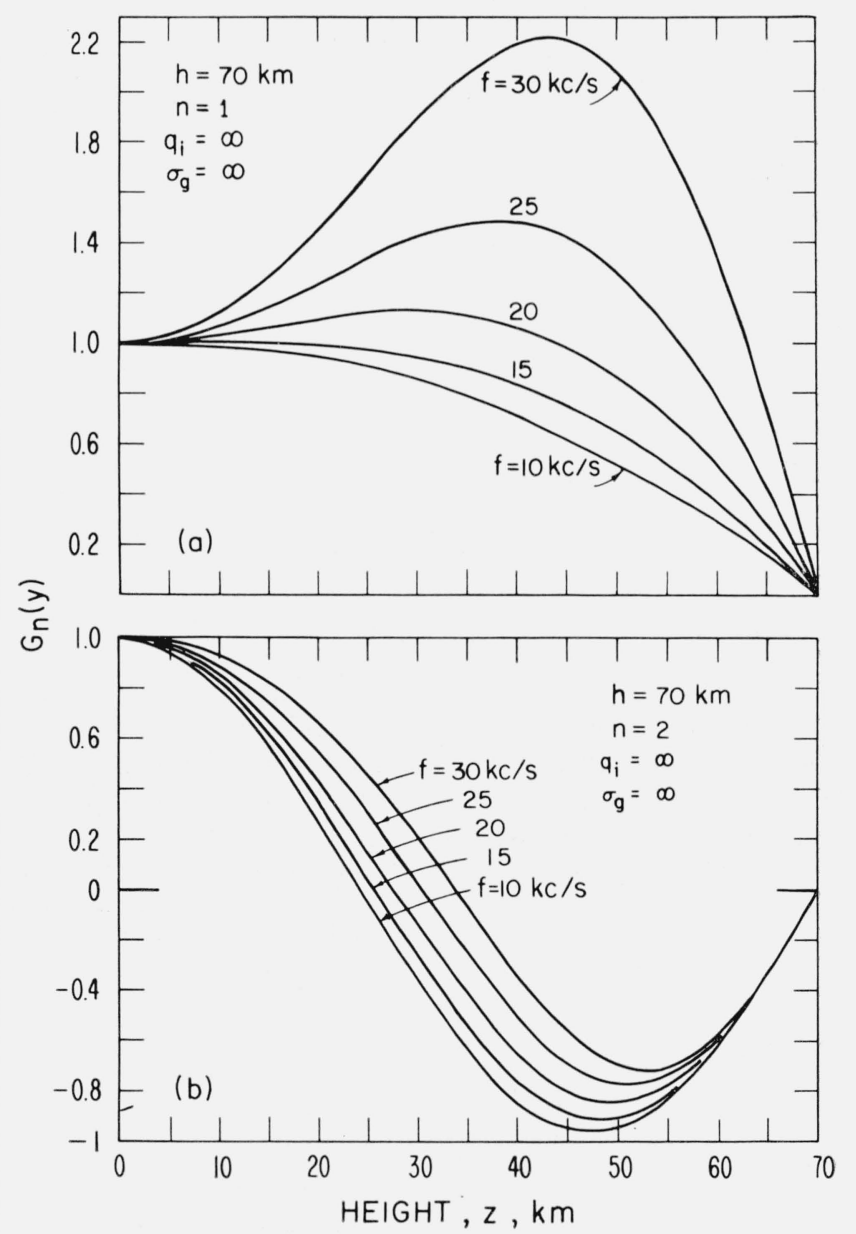

Figures $3 \mathrm{a}$ and $3 \mathrm{~b}$. The height-gain functions for the first and second order modes for an earth-ionosphere waveguide with perfectly reflecting boundaries.

In this case $G_{n}(y)$ is real. In these curves the reflecting height is fixed at $70 \mathrm{~km}$. 


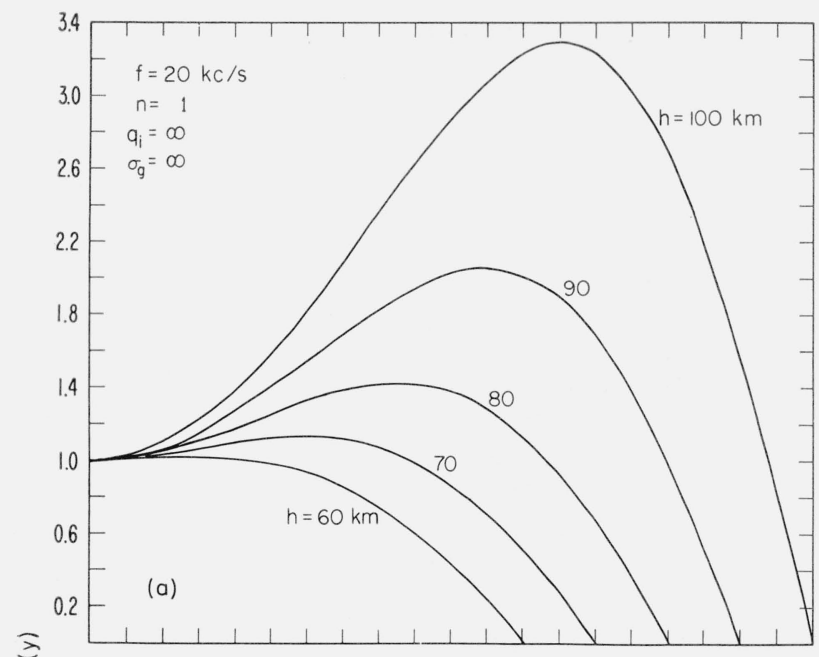

든

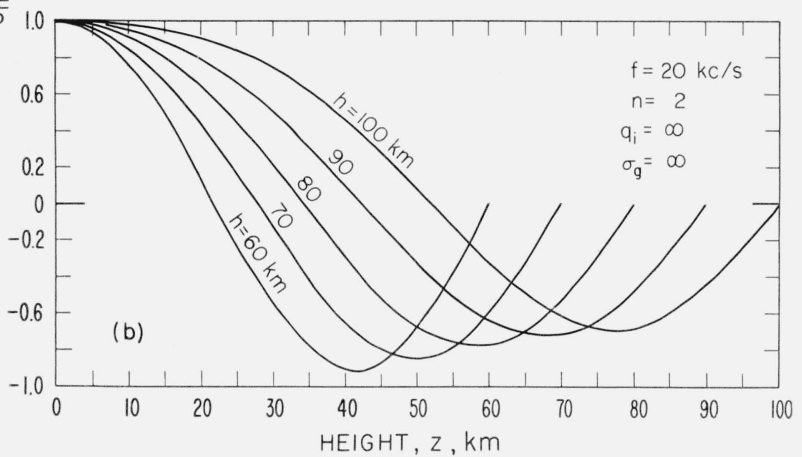

Figures $4 \mathrm{a}$ and $4 \mathrm{~b}$. The height-gain functions for the first and second order modes for an earth-ionosphere waveguide with perfectly reflecting boundary.

In these curves the frequency is fixed at $20 \mathrm{kc} / \mathrm{s}$.

is finite at the upper boundary and the phase is of the order of $-90^{\circ}$. Such a behavior is compatible with the boundary condition at the sharply bounded ionized layer.

It is seen"from the curves (e.g., figures $3 \mathrm{a}, 4 \mathrm{a}, 5 \mathrm{a}$, and 6 a) that the field associated with the first order mode rises to a maximum at a level below the reflecting layer. In this sense the mode is analogous to Lord Rayleigh's whispering gallery (acoustical) mode which he observed on the inside of the dome of St. Paul's cathedral [Rayleigh, 1910; Budden, 1962]. When dealing with the earth-ionosphere waveguide it is appropriate to call this the earth-detached mode since it depends only very slightly on ground conductivity [Wait, 1962; Spies and Wait, 1961]. It may be observed that the form of the height variation is generally quite insensitive to the electrical characteristics of the reflecting boundaries.

The effect of finite ground conductivity is seen by comparing figures $5 \mathrm{a}$ and $5 \mathrm{~b}$ for $\sigma_{g}=\infty$ with figures $6 \mathrm{a}$ and $6 \mathrm{~b}$ for $\sigma_{g}=5 \times 10^{-3}$. It is evident that the finite conductivity of the lower boundary modifies the amplitude of $G_{n}(y)$ only slightly although the phase is noticeably affected. This behavior is con-
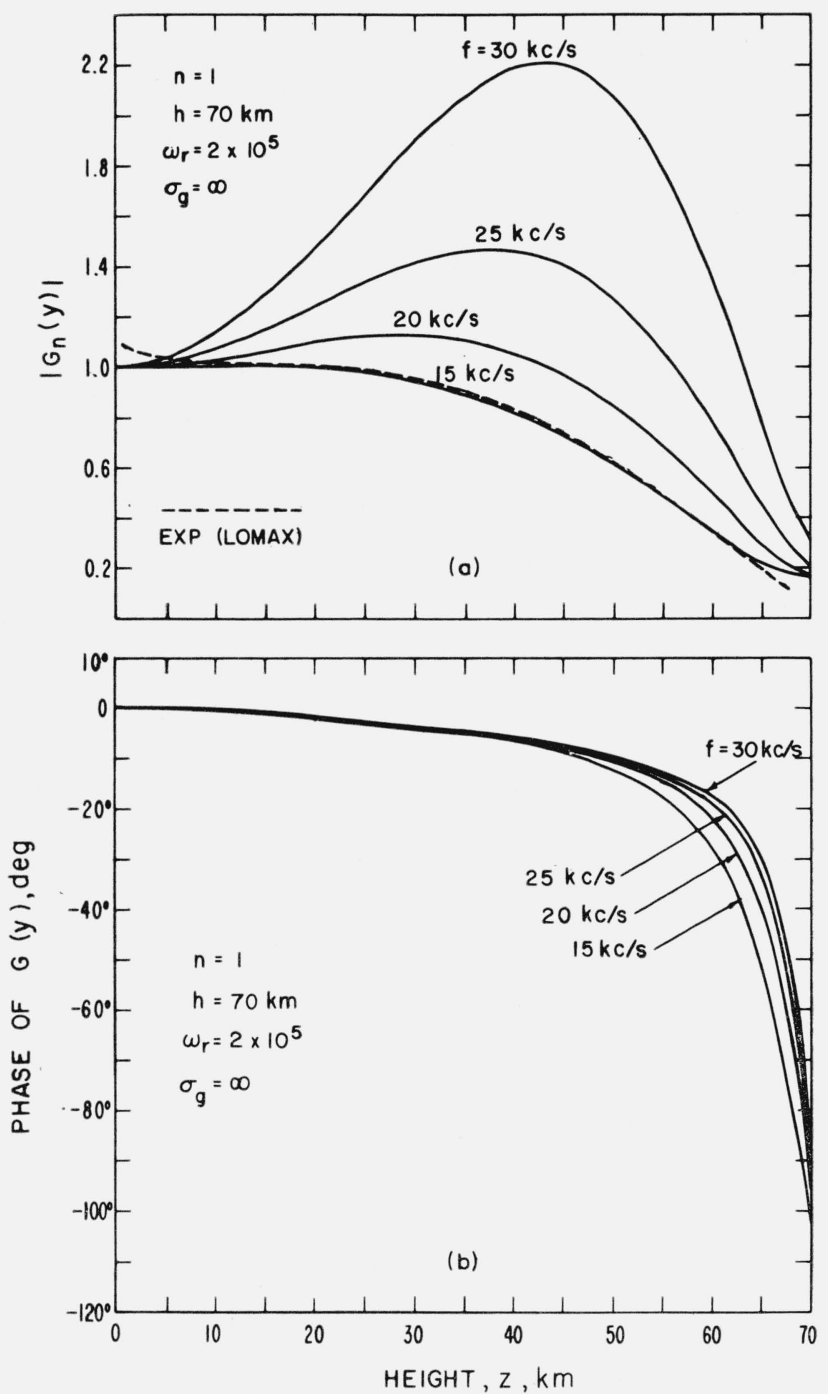

Figures 5a and 5b. The amplitude and phase of the heightgain function for an imperfectly reflecting upper layer and a perfectly conducting lower boundary.

The dashed curve in (a) is based on a rocket measurement at $18.0 \mathrm{kc} / \mathrm{s}$ during daytime for a path over sea water.

sistent with the series development for height-gain functions given previously [Wait, 1962]. In particular, it was indicated that, for low heights, the finite conductivity tends to reduce $\left|G_{n}(y)\right|$ below unity and the phase of $G_{n}(y)$ is positive by a small amount. For propagation over sea water it is permissible to regard $\sigma_{g}$ as being infinite.

As mentioned in the introduction, the height-gain functions should describe the variation of the field measured in a rocket as it proceeds from the ground up to the ionosphere. Such an experiment has actually been carried out by Lomax [1961] at Eglin Air Force Base in Florida. He employed the 18.0 $\mathrm{kc} / \mathrm{s}$ transmission from station NBA in the Canal Zone, Panama. At this distance, in the daytime, it is expected the first-order mode predominates. 

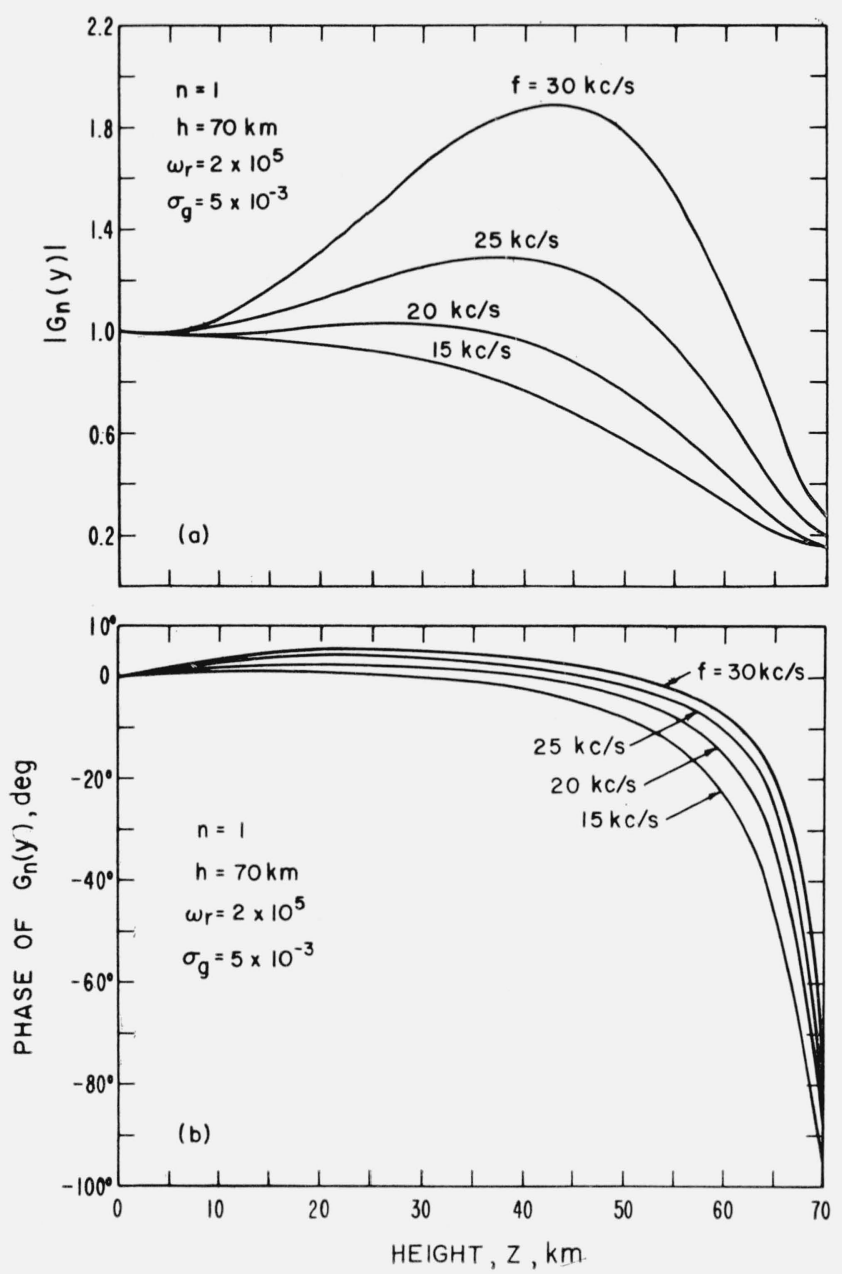

Figures $6 \mathrm{a}$ and $6 \mathrm{~b}$. The amplitude and phase of the heightgain function for an imperfectly reflecting upper layer and a finitely conducting lower layer (typical of propagation over land).

A sketch of Lomax's results for the vertical electrical field is shown in figure 5a. It follows the theoretical curve for $15 \mathrm{kc} / \mathrm{s}$ quite closely. Over the range of heights from 2.5 to $60 \mathrm{~km}$ Lomax estimates that the signal strength as plotted is accurate to within $2 \mathrm{db}$. The slight increase at very low heights has been attributed to the exhaust plume which has the effect of increasing the effective length of the receiving antenna. At great heights there were also some departures between the calculated and experimental curves, but here the experimental data is not considered to be reliable.

\section{Concluding Remarks}

The curves given in this paper, although based on an idealized model, should provide preliminary information concerning the behavior of the VLF field at large heights in the atmosphere. Contrary to commonly accepted ideas the field may increase by a significant amount as the transmitting or receiving antennas are raised. The effect should be particularly noticeable at the upper end of the VLF band at long ranges.

It might be mentioned that the results in this paper, for the height-gain functions, refer specifically to the vertical electric field. However, to a very good approximation they also hold for the horizontal magnetic field component. On the other hand, the horizontal electric field component which was not discussed in this paper actually is proportional to the derivative with respect to height of the function $G_{n}(y)$. Thus, we can expect the horizontal electric field to be very small on the ground and at low heights. However, at heights above $30 \mathrm{~km}$ the horizontal field may be quite large.

I thank Mrs. L. C. Walters and Mrs. C. M. Jackson for their assistance with the computations, Mr. V. Pfannenstiel who drafted the illustrations and, finally, Mrs. Eileen Brackett for help in preparing the manuscript.

The work has been supported by the Advanced Research Projects Agency, Washington, D.C., Order No. 183-62.

\section{References}

Budden, K. G., (1962), The waveguide mode theory of wave propagation (Prentice-Hall, New York).

Lomax, J. B., (June 1961), Measurement of VLF transmission characteristics of the ionosphere with instrumented NikeCajun Rockets, final report from Stanford Research Institute on Contract NOw $60-0405$ to the U.S. Navy.

Rayleigh, Lord, (1910), The problem of the whispering gallery, Phil. Mag. 20, 1001. [See also 27, 100 (1914)].

Spies, K. P., and J. R., Wait (July 17, 1961), Mode calculations for VLF propagation in the earth-ionosphere waveguide, NBS Tech. Note No. 114

Wait, J. R., (Jan.-Feb. 1961), A new approach to the mode theory of VLF propagation, J. Research NBS 65D (Radio Prop.), No. 1, 37-46.

Wait, J. R. (1962). Chapter VII, Electromagnetic waves in stratified media (Pergamon Press, Oxford).

(Paper 67D2-254) 